\title{
A Review on Matching For Sketch Technique
}

\author{
Prasad Mahale ${ }^{1}$, Prof. Ms. Deepshikha Patel ${ }^{2}$, Prof Dr. Bhupen Gaur ${ }^{3}$ \\ ${ }^{1,2}$ (Information Technology, TIT Bhopal, India) \\ ${ }^{3}$ (Computer Science, TIT Bhopal, India)
}

\begin{abstract}
In the area of digital image processing content based image retrieval (CBIR) is one of the most popular, researches raising area. Many tools like Google, Yahoo \& other used textual annotation of images. The principle work that was done by CBIR is to extract visual content of image intuitively like color, shape \& texture. Additionally sketch based image retrieval system (SBIR) is also plays very imperative role within the content based image retrieval system (CBIR). The system including problem \& challenges concerned with design and constitution of content based image retrieval system. The vital objective that holds the information gap between a sketch \& color image we have to design \& appliance task specific descriptor. The system states that a description about a possible solution for the implementation of descriptor to carry a special sequence of altering the steps of the reconstruction of full color image sketch can be compared. For the preparation of gallery can take a shop images with possible application for forensic sketch identical images.
\end{abstract}

Keywords: Sketch-based image retrieval, Image databases, Image descriptors, CBIR, SBIR.

\section{Introduction}

Data representing a two dimensional scenes. A digital image is composed of pixels arranged in rectangular way of array with a certain height $\&$ width. Each pixel may consist of one or more bits of information, representing brightness of images at possibly including color information needed as RGB, to the event performing analysis of picture using techniques that can identify shades, colors \& relationship that cannot be perceived by the human eyes. The fundamental of image processing that can be used to solve identification problems, such as in forensic medicine or in creating weather maps from satellite such as refining a picture in a scanned or entered from video source.

For the escalation of information technology an extensive number of data had to be maintain, growth \& stored. It contains also the textual and the visual information. Similarly the presence \& quick enlargement of the system and maximum corresponding measure of data have to be maintained. It is very essential term to searching the adaptability of the information ser. The maximum growth of data maintains \& transformation of internet had to be change the system. When we search appropriate image we cannot apply a dynamic methods.

But in case of text it is very simple to find with the reference of keywords. It is human ability to remember \& recall visual information more efficiently by using an example of various objects, colors \& shapes with possible evaluation of the architecture of image. Sometime want to search a data when considering many cases it is necessary to be recall. For accurate image search sometimes have to follow some features of image \& these features considered as a keyword. The human is a displaying some textures, we look for architecture using other shapes \& follow this access also on the basis of classifying images. The system goal is to construct belongs to content based image retrieval, which will be fetch using sketches in frequently used database. For the basis of reclaim method the system provides the separate drawing area, where we can draw different sketches.

\section{Literature Review}

D. Comaniciu and P. Meer. [1], introduced the performance for the recovery of significance we are presenting image featured. The system explains the techniques that explain related to mean shift algorithm (MSA) to measures the density gradients with simple non metric procedure. To overcome the robust clustering through paper color segmentation is proposed. The segmentation is completely self determining become when the user consider only class. For the evaluation of content based query system it must ensure that the same program can generate high quality edge image, provide by separating representative color. To evaluate a sound workstation we have to consider $512 * 512$ color image. The sample considers a gray level image to handle only glow equalized. The classicality of basic enumerate module with the reference of mean shift algorithm for realization of the pull of to test feature space analysis very fast. It helps with Ultra SPARC 1 workstation to spectrum from $512 * 512$ pixel images into 10-20 feature can be evaluated in less than $10 \mathrm{sec}$. The further result of cleavage can be strain in the image region by local processing.

D.G. Lowe [2] the advance class of local image features that uses by an object recognition system. The affection are consistent to image scaling, rotation, translation fractionally invariant to enlightening changes \& 
affine or projection that having 3D features with neurons is in bottom temporal cortex that are used for object allowance in elementary perception \& these features shares equivalent properties. Features are adequately ascertained through a various steps filtering accession that visualize static points in extent of capacity. To delegation of representation the blurred picture gradients in multiple collective exertion planes at various scales with image keys are generated that possible for local geometric anamorphosis. In this system keys are used to nearest resident indexing steps that analyze candidate object corresponding matches. This system states the proposed result shows us that the robust observation of objects can be conclude in rearrangement a partially congest image with a calculating time under 2 sec.

N. Dalal and B. Triggs [3] the technique states that existence of gradient orient restricted portion of an image. The system explains their algorithm on the basis of the problem of pedestrian detection in the static image. Since they are increase their evaluation module includes human detection film \& video. The description by the edge indication or the dispensation of extent gradients with local image object visualization $\&$ shape, it is magnificent thought behind HOG descriptor. We can justify this algorithm on the basis of to dividing image into small linked region, called cells \& after for each cell arrange a histogram of gradient direction for pixel within the cell for the representation of descriptor. To evaluate or measuring a more accuracy the local normalized by manipulating a measure intensity across a larger region of image called block.

There are some factor are very important for having good result we evaluate with same factor being considered \& those are having a high quality local contrast normalization in overlapping descriptor blocks relatively coarse spatial binning, fine orientation binning, concluding throughout fine scale gradient the impact of each stage of estimation of performance should be considered. The newly access gives near perfect compilation on original MIT pedestrian database, so in paper propose a more demanding dataset containing thousands commented large range of pose disparity \& background.

T. Deselaers, D. Keysers, and H. Ney. [4] Design the experimental comparison of huge number of separate image descriptor for content based image retrieval is established. There are many systems that have different new technique \& descriptor for content based image retrieval extracting their newly proposed methods as most efficiency in-depth comparison with all current methods that established nowadays. This system proposed initially a presentation of large variety of features for the content based image retrieval system \& distinguish them quantifiable on different task: personal photo retrieval, medical image retrieval, building retrieval \& stock photo retrieval. On the basis of this system taking into consideration five different publicity available image databases are used $\&$ the retrieval evaluation feature performed in detail. The concept concludes with endorsement which feature is good performing for data, which nominate for simple \& efficient method to find initial steps of idea features for a new task of statements. And again the additional activity the correlation of feature is evaluate which states the concern way of simple \& intuitive structure to examine the primary set of features for the new task.

A.K. Jain, J.E. Lee, R. Jin, and N. Gregg [5] the system concludes that generous in most civil neighborhood and present exasperation $\&$ as disgrace. Still the arm of law system have searching $\&$ they found them to be useful for the sympathize a gang activities, and expose the vicinity of gang province enlarge metropolitan areas. The current method for equivalent and recover graffiti is based on standers database search that is not only imprecise but also time captivity. The system proposed the CBIR system for automatic analogues \& retrieval of the images for graffiti. The system enact each image of graffiti by package of SIFT features. The harmony of agreement between the query image \& graffiti image is compared based on the various umber of same SIFT feature between the two image under static geometric constraint in database. The experimental result shows the two graffiti database with various thousands of images shows supporting and encouraging result.

A.K. Jain, J.E. Lee, R. Jin, and N. Gregg [6] this system proposed the evaluation of the traditionally collected human body tattoo images and consistently used a law enforcement $t$ examine the victim $\&$ suspect verification. Then the combination of tattoos as same the current practices based on some keywords. The content based image retrieval has developed for database of tattoo images. This system separates on the concept of SIFT for the basis image based retrieval. The keywords that use in paper belongs to individual images is both time some \& illusory. To improve the retrieval time \& accuracy it utilizes with both body location of tattoo and tattoo classes. In SIFT key points matching database false retrieval we are also announce the geometrical constraints. In all respective experimental result on thousands concern against an operational database over more than 60000 tattoo images is displayed a rank-20 variety of good average matching time per query is also good.

The system represents a CBIR system for the tattoo images. The system terminologies having great values in capturing \& pretend the suspect \& identification of victims in the forensic and arm of the law application.

A.K. Jain, J.E. Lee, and R. Jin [7] the system implements a local feature based at various method for matching a facial sketch Images to the facial photo picks, it holds firstly feature based matching. Take a photo correspondence (photo \& sketch based on same issue) has ability to match a sketch photo. It also enhance the 
first features by using SIFT descriptor, again we use a distance transform to make a bridge between sketch \& photo for the common representation with similar feature adaption. And it possible to o consider by fusing previous both methods. In direct matching technique we are simply measure the distance of SIFT descriptor between sketches \& photos. Further recognition improvements are shown by score level with fusion of two sketch matches. Improve performance by feature based methods. Turk \& Pent lands Holistic Eigen face matching algorithm served as precedence for modern face recognition engines. This has promoted researches to turn the focus of their research in face recognition to more difficult problems such as varying illumination, nonfrontal pose \& occlusion. The proposed method differ significance, in that we use a local feature-based representation to compare sketches \& photos. Previous methods are based on holistic matching on sketches that for transferring a photograph either using linear transformation directly on the intensity images or by generating a synthesis photograph. The feature based matcher it used in conjunction with aforementioned holistic algorithm for hybrid matching

M. Eitz, K. Hildebrand, T. Boubekeur, and M. Alexa [8] address the problem of fast, large scale based image retrieval with searching over one million images in a database. The proposed retrieval method do not range well belongs largely creates database it the context of associatively surprised search \& nominate two different methods for the optionally examines that the significantly exceed current approach. The system defines the descriptor that explains the construction such that both full color image \& range undergo properly save processing steps. First of all system searches the similar manipulating structure image evaluating gradient orientation. Then from the best filtering \& matching image have been clustered based on leading distribution of colors to neutralize the lack of color based decision during the primary search. So from situated method being examined $\&$ after that the overall query results manifest that the system offers. Perceptive access to large image database user friendly sketch \& brows interface.

R. Hu, M. Barnard, and J. Collomosse [5] the system represents the retrieval system manipulating with free hand sketches with queries of depicted shape. For the evaluation of characterize shape it must be initialized with gradient field HOG (GF-HOG) at image descriptor as illustrate invariant images. For the measurement of better codebook base retrieval \& formation of local spatial structure with various sketches can be efficiently related. It seems to be a various review of improved retrieved accuracy over 3 ever 3 consisted leading descriptor (self similarity, SIFT, HOG) with reference of two databases (Flickr160, ETHZ extended object) \& after we look for further concept that how it work with combination of GF-HOG \& RNSAC for the relevant images to localized sketched objects. And here also extract the sketch driven of photo montage application based on this system.

The all corresponding consideration shows us that the proposed system GF-HOG can be very efficient \& system terminologies are incorporated into BoW system. For the representation of sketch based image retrieval and the paper states that BoW system is not previously used to retrieval system. This system also demonstrates a prototype application for the purpose of retrieval technique.

\section{Discussion And Problems}

The above discussed techniques are providing the superior result but they are also lacking in certain things. This technique to generate the good result takes a time from few minutes to the hours. If we figure the size, shape \& textures of the image to recover in that case some of the techniques unable to produce the good result, because some of the techniques are designed for specific database and having some limitations. But they appointed some extra methods are success to fulfill the new inventions, and likely to be proposed new methods. Present authors explain the specific area like SIFT, human detection, image retrieval, tattoo images etc. some techniques follow few database to check random result, that current procedure are working or not, otherwise likely have to consider more than 4 database to evaluate proper result.

\section{Table}

Table 1 List of authors that contribute their valuable ideas of sketches.

\begin{tabular}{|c|c|c|c|}
\hline Sr.No. & Author & Proposed Method & Year \\
\hline 1 & D. Comaniciu, and P. Meer & Robust analysis color image segmentation & 1997 \\
\hline 2 & D.G. Lowe & SIFT feature transform & 1999 \\
\hline 3 & M. Elad & Evaluation of descriptor for specified images & 2005 \\
\hline 4 & N. Dalal, and B. Triggs & Normalized histogram \\
of gradient orientations & 2005 \\
\hline 5 & $\begin{array}{c}\text { T. Deselaers, D. Keysers, and } \\
\text { H. Ney }\end{array}$ & Comparison of Images features with CBIR & 2007 \\
\hline 6 & A.K. Jain, J.E. Lee & Graffiti matching retrieval system & 2009 \\
\hline 7 & A.K. Jain, J.E. Lee & Tattoo images with CBIR in law enforcement & 2009 \\
\hline 8 & M. Eitz, K. Hildebrand & Extraction of descriptor & 2010 \\
\hline
\end{tabular}




\section{Conclusion}

A presentation of sketch-based image retrieval system through the goals of this paper performed to composition, conceive and examine. The consideration of retrieval process has to be individualistic and deeply reciprocal and it is very consistent to extract the features of the image that based on sketches for matching a techniques. The robustness or concentrated of the method is essential in some amount of turbulence, which capable also be in state of few sample images. The already implement drawn image without adaptation cannot be analyzed with other color image, or its bound consideration. A different distance convert step was imported.

There are various methods are proposed to evaluate efficient technique, likewise it concludes that the system based on specific database. The presentation gives us detail information throughout image sketch to the matching technique of given parameters. The search result list is clarified using the SIFT-based multi-level clarification. It states that division of retrieval acknowledgement a considerable decision possibility was taken by user on that statement, and for that he can select from bunch of results. It was extracted by examining a given database and there are several database are used. The match technique is evaluate by means of the images are including in database, so it carries more than 10000 images to matching purpose. From this detailed survey we discussed the advantages and shortcomings of all image sketching techniques. This algorithm works for texture as well as structure synthesis. But this work with maximum accuracy when regions contains simple texture, shapes \& structure. Overall study tells that all technique trying to provide better result in terms of match from sketch and also trying to improve the efficiency in terms of the image clustering algorithm and other techniques.

\section{References}

[1] D. Comaniciu, and P. Meer, "Robust analysis of feature spaces: color image segmentation," IEEE Conference on Computer Vision and Pattern Recognition, pp. 750-755, June 1997.

[2] D.G. Lowe, “Object Recognition from Local Scale-Invariant Features,” IEEE International Conference on Computer Vision, vol. 2, p. $1150,1999$.

[3] N. Dalal, and B. Triggs, "Histograms of oriented gradients for human detection," IEEE Conference on Computer Vision and Pattern Recognition, pp. 886-893, July 2005.

[4] T. Deselaers, D. Keysers, and H. Ney, "Features for image retrieval: an experimental comparison," Information Retrieval, vol. 11, pp. 77-107, December 2007.

[5] A.K. Jain, J.E. Lee, R. Jin, and N. Gregg, “Graffiti-ID: matching retrieval of graffiti images,” ACM MM, MiFor'09, pp. 1-6, 2009.

[6] A.K. Jain, J.E. Lee, R. Jin, and N. Gregg, "Content based image retrieval: an application to tattoo images," IEEE International Conference on Image Processing, pp. 2745-2748, November 2009.

[7] A.K. Jain, J.E. Lee, and R. Jin, "Sketch to photo matching: a feature-based approach," Proc. SPIE, Biometric Technology for Human Identification VII, vol. 7667, pp. 766702-766702, 2010.

[8] M. Eitz, K. Hildebrand, T. Boubekeur, and M. Alexa, “An evaluation of descriptors for large-scale image retrieval from sketched feature lines," Computers and Graphics, vol. 34, pp. 482-498, October 2010. 